\title{
ISOMORPHISMS BETWEEN SEMIGROUPS OF INTEGERS
}

\section{PAGE PAINTER}

1. Introduction. Let $j$ and $k$ be positive integers. Let $G_{j}$ be the multiplicative group of residue classes mod $j$ whose elements are relatively prime to $j$, and denote its elements by $R_{1}^{\prime}, R_{2}^{\prime}, \cdots, R_{\phi(j)}^{\prime}$. We will always identify $R_{1}^{\prime}$ with the identity element, the class of all in tegers congruent to $1 \bmod j$. Let $R_{i}$ be the set of positive integers in $R_{i}^{\prime}$, and note that $R_{1}$ is a semigroup under multiplication. Similarly $G_{k}$ has elements $S_{1}^{\prime}, S_{2}^{\prime}, \cdots, S_{\phi(k)}^{\prime}$ where $S_{i}$ is the set of positive integers in $S_{i}^{\prime}$. Again $S_{1}$ is a semigroup.

For the case $j=1$ and $k=2$, any one-to-one mapping of the primes onto the odd primes can be extended to an isomorphism of $R_{1}$ $=\{1,2,3, \cdots\}$ on to $S_{1}=\{1,3,5, \cdots\}$. However, the semigroup $\{1,4,7, \ldots\}$ corresponding to the identity element of $G_{3}$ is not isomorphic to the above semigroups since unique factorization holds in the former cases but not in the latter $(100=10 \cdot 10=25 \cdot 4)$. Our main result is that $G_{j}$ is isomorphic to $G_{k}$ (written $G_{j} \sim G_{k}$ ) if and only if $R_{1} \sim S_{1}$. The problem of the existence of isomorphisms between these infinite semigroups is thereby reduced to a solvable problem.

\section{Isomorphisms.}

Theorem. $G_{j} \sim G_{k}$ if and only if $R_{1} \sim S_{1}$.

Proof. Assume $G_{j} \sim G_{k}$. We may choose our notation so that the class $S_{i}^{\prime}$ is the image of the class $R_{i}^{\prime}$. Thus the isomorphism induces a mapping $\alpha$ such that $\alpha\left(R_{i}\right)=S_{i}$. We use $\alpha$ to define a mapping of the integers in $R_{1}$ on to the integers in $S_{1}$ as follows: for each $i=1,2, \cdots$, $\phi(j)$ set up a one-to-one correspondence between the primes in $R_{i}$ and the primes in $S_{i}$. Now given any element $a$ in $R_{1}$, write its decomposition into prime factors $p_{1} p_{2} p_{3} \ldots$ (these factors must be in the sets $R_{i}$ ), and map these primes onto their corresponding primes $q_{1}, q_{2}$, $q_{3}, \cdots$ in the sets $S_{i}$. In this way we define a mapping $a \rightarrow q_{1} q_{2} q_{3} \cdots$ It is easy to show that this gives an isomorphism of $R_{1}$ onto $S_{1}$.

Before proving the converse, we note that if $F_{j}=\left\{H_{1}^{\prime}, H_{2}^{\prime}, \cdots\right\}$ is a subgroup of $G_{j}$, where $H_{i}$ is again the set of positive integers in $H_{i}^{\prime}$, then $H=H_{1} \cup H_{2} \cup \ldots$ is a semigroup under multiplication. Let $F_{k}=\left\{I_{1}^{\prime}, I_{2}^{\prime}, \cdots\right\}$ be a subgroup of $G_{k}$, and let $I=I_{1} \cup I_{2} \cup \cdots$. The above proof is easily generalized to show that if $G_{j} \sim G_{k}$ and $F_{j} \sim F_{k}$, then $H \sim I$. The converse is false since it is always true that

Received by the editors March 30, 1966. 
$R_{1} \cup R_{2} \cup \ldots \cup R_{\phi(j)} \sim S_{1} \cup S_{2} \cup \ldots \cup S_{\phi(k)}$ (unique factorization holds in both semigroups).

Conversely assume $R_{1} \sim S_{1}$. Let $p$ be any prime such that $(p, j)=1$, and let $t$ be the least positive integer such that $p^{t} \equiv 1(\bmod j)$. Then $p^{t}$ is a member of $R_{1}$, and its image under the $R_{1}$ to $S_{1}$ isomorphism is some product of primes $q_{1} q_{2} \cdots q_{n}$. Let $v$ be the least positive integer such that $q_{1}^{0} \equiv 1(\bmod k)$. Under the $R_{1}$ to $S_{1}$ isomorphism $p^{t v}$ maps onto $\left(q_{1} q_{2} \cdots q_{n}\right)^{v}=q_{1}^{0}\left(q_{2} \cdots q_{n}\right)^{v}$. Since the only factorization of $p^{t v}$ in to irreducible elements of $R_{1}$ contains $v$ irreducible factors each $p^{t},\left(q_{1} q_{2} \cdots q_{n}\right)^{v}$ must factor uniquely into $v$ identical factors in $S_{1}$. But $\left(q_{1} q_{2} \cdots q_{n}\right)^{v}$ has an irreducible factor $q_{1}^{0}$ in $S_{1}$, so we conclude that $p^{t}$ maps onto $q_{1}^{0}$. Furthermore all the $q_{i}$ are equal and $n=v$.

Now let $p_{0}$ be another prime, different from $p$, such that $p_{0} \equiv p$ $(\bmod j)$. Then $t$ is the least positive integer such that $p_{0}^{t} \equiv 1(\bmod j)$, and under the $R_{1}$ to $S_{1}$ isomorphism $p_{0}^{t}$ maps onto $q_{0}^{w}$, where $q_{0}$ is a prime different from $q_{1}$. Consequently $\left(p p_{0}^{t-1}\right)^{t}$ maps on to $q_{1}^{0} q_{1}^{w(t-1)}$. Since this expression must be a $t$ th power, we have $t \mid v$. Similarly we can show that $v \mid t$, so that $t=v$.

We now see that the $R_{1}$ to $S_{1}$ isomorphism can be embedded in a larger mapping $\psi$ by defining $\psi(p)=q_{1}, \psi\left(p_{0}\right)=q_{0}$ : similarly every prime $p_{i}$ (not a divisor of $j$ ) maps on to a prime $q_{i}$ (not a divisor of $k$ ), where the $R_{1}$ to $S_{1}$ isomorphism maps $p_{i}^{\phi(j)}$ onto $q_{i}^{\phi(j)}$. If $a$ is any positive integer relatively prime to $j$, write its decomposition in to prime factors $p_{1} p_{2} p_{3} \ldots$, and define $\psi(a)=\psi\left(p_{1}\right) \psi\left(p_{2}\right) \psi\left(p_{3}\right) \cdots$.

Let $a$ and $b$ be elements of $R_{i}$. Then both $a b^{\phi(j)-1}$ and $b^{\phi(j)}$ are in $R_{1}$. Since $\psi$, restricted to $R_{1}$, is the $R_{1}$ to $S_{1}$ isomorphism, both of these products are mapped by $\psi$ into $S_{1}$. Now it is easy to show that $\psi(a)$ and $\psi(b)$ are in the same set $S_{i}(\psi$ preserves congruence), and that $\psi$ maps $R_{\boldsymbol{i}}$ onto $S_{i}$. Hence we can define a mapping of $G_{j}$ onto $G_{k}$ by defining the image of $R_{i}^{\prime}$ to be $S_{i}^{\prime}$, where $\psi$ maps $R_{i}$ onto $S_{i}$. It is easy to verify that this gives an isomorphism of $G_{j}$ onto $G_{k}$.

3. Monomorphisms and epihomomorphisms. We can always define an isomorphism of $R_{1}$ in to $S_{1}$ by setting up a one-to-one mapping of the primes into the primes of $S_{1}$ and extending the mapping to the product of primes as before. By defining a mapping of the primes in $R_{1}$ onto $S_{1}$ and by extending this mapping so that any prime not in $R_{1}$ maps onto 1 , we have a homomorphism of $R_{1}$ on to $S_{1}$. However, there are many examples where there is no isomorphism of $G_{j}$ in to $G_{k}$ and where there is no homomorphism of $G_{j}$ on to $G_{k}$ e.g. $G_{5}$ and $G_{8}$. Hence the theorem cannot be generalized to the case of monomorphisms or to the case of epihomomorphisms. 